\title{
Transport on randomly evolving trees
}

\author{
L. Pál \\ KFKI Atomic Energy Research Institute, H-1525 Budapest 114, POB 49, Hungary
}

\begin{abstract}
The time process of transport on randomly evolving trees is investigated. By introducing the notions of living and dead nodes, a model of random tree evolution is constructed which describes the spreading in time of objects corresponding to nodes. It is assumed that at $t=0$ the tree consists of a single living node (root), from which the evolution may begin. At a certain time instant $\tau \geq 0$, the root produces $\nu \geq 0$ living nodes connected by lines to the root which becomes dead at the moment of the offspring production. In the evolution process each of the new living nodes evolves further like a root independently of the others. By using the methods of the agedependent branching processes we derive the joint distribution function of the numbers of living and dead nodes, and determine the correlation between these node numbers as a function of time. It is proved that the correlation function converges to $\sqrt{3} / 2$ independently of the distributions of $\nu$ and $\tau$ when $q_{1} \rightarrow 1$ and $t \rightarrow \infty$. Also analyzed are the stochastic properties of the end-nodes; and the correlation between the numbers of living and dead end-nodes is shown to change its character suddenly at the very beginning of the evolution process. The survival probability of random trees is investigated and expressions are derived for this probability.
\end{abstract}

PACS numbers: $05.40 . \mathrm{Ca}, 02.50 . \mathrm{Cw}, 05.10 . \mathrm{Gg}$

\section{INTRODUCTION}

Recently there has been a growing interest in randomly evolving graphs since a large number of practically important problems can be analyzed in this way. In the last few years so many papers have been presented in this field that any list of references would be far from complete by any standards. It is fortunate that two outstanding review papers 1], 2] have been published in 2002. Thus the author of the present paper does not feel obliged to cite the very large amount of important references. In addition, one must mention the excellent book by Dorogovtsev and Mendes [3], which gives a very interesting summary of the actual problems of the growth and structure of random networks.

It has been long known that the random evolution of a tree ${ }^{1}$ corresponds to a Galton-Watson process [4] which describes the formation of a population which starts at $t=0$ with one individual and in which at discrete time moments $t=1,2, \ldots$ each individual produces a random number of offspring with the same distribution independently of the others. There are many interesting papers on this subject, statements of which are analyzed and generalized in Ref. 5]. The purpose of this paper is to study the random tree evolution with continuous time parameter with the hope that this may help to a better understanding of the nature of the age-dependent branching processes which play a significant role in many fields of physical and biological sciences [6, 7, 8, 19, 10, 11, 12, 13]. The general theory of age-dependent branching processes is excellently summarized in Ref. [4] and in Ref. [15], therefore we decided to focus only on the correlation and survival properties of the randomly evolving trees.

The paper is organized as follows. In Sec. II the exact description of the random process is given, while in Sec. III the derivations of the basic equations for the generating functions can be found. The properties of average characteristics (expectation values, variances, and correlation functions) are discussed in Sec. IV. Survival probability of trees is determined in Sec. V, and finally, the conclusions are summarized in Sec. VI. It is to mention that for more detailed analysis of the problem see [14].

\section{DESCRIPTION OF THE RANDOM PROCESS}

We will study the random evolution of trees which consist of living and dead nodes as well as lines connecting the nodes. A living node is unstable, and after a certain time $\tau$ may become dead. The lifetime $\tau$ is a random variable, and $\mathcal{P}\{\tau \in(t, t+d t)\}=d T(t)$ denotes the probability of finding $\tau$ in the time interval $(t, t+d t)$. A dead node is inactive, it cannot change its state, and is unable to interact with the other nodes.

The evolution process can be described as follows. Let us suppose that at time instance $t=0$ the tree consists of a single living node called the root and let $\mathcal{S}_{0}$ denote this state of the tree. At the end of its life the root creates

\footnotetext{
1 A randomly evolving tree is a connected graph containing no cycles, and growing from a single node (root) according to well-defined rules.
} 
$\nu=0,1,2, \ldots$ new living nodes and immediately after that it becomes dead. The number $\nu$ of the offspring is a random variable, and the probabilities $\mathcal{P}\{\nu=k\}=f_{k}, \quad k=0,1,2, \ldots$ are assumed to be known. The new living nodes are promptly connected to the dead one and each of them can evolve further like a root independently of the others. This means that the tree from its initial state $\mathcal{S}_{0}$ may evolve further by two mutually exclusive ways, in which the root either

- survives, and thus the tree remains in its initial state during the whole time interval $(0, t)$, or

- the root does die in an infinitesimally small subinterval $\left(t^{\prime}, t^{\prime}+d t^{\prime}\right) \in(0, t)$ and produces $j \geq 0$ new living nodes. Each of these nodes will evolve further in the remaining time interval $\left(t^{\prime}, t\right)$ independently of the others like the root.

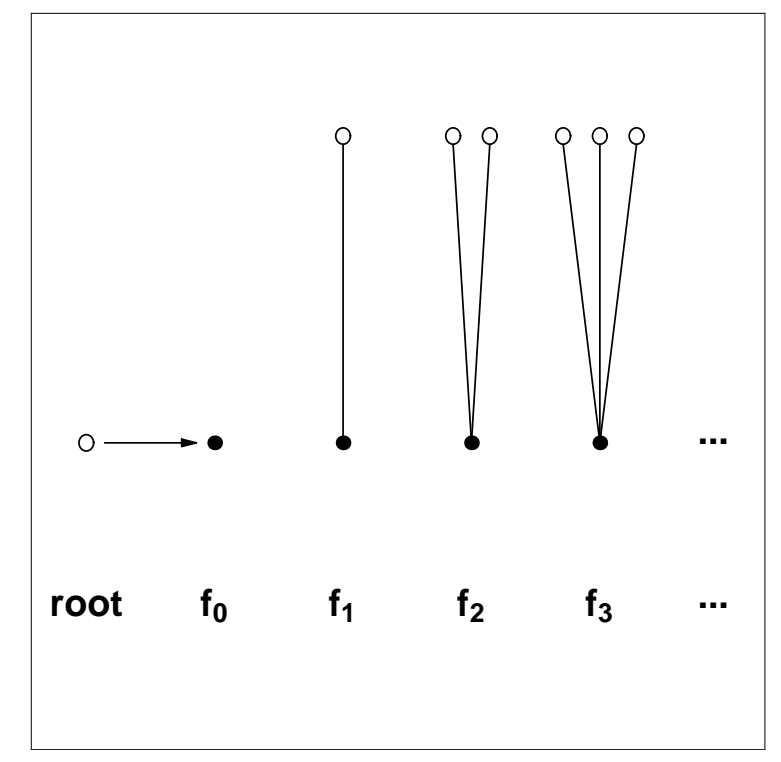

FIG. 1: First step of the tree evolution. The new state arises from the initial state which consists of a single root. Four of the possible new states are illustrated in the figure. Living nodes are denoted by white circles and the dead ones by black circles. $f_{0}, f_{1}, f_{2}, f_{3}, \ldots$ are the probabilities of producing $0,1,2,3, \ldots$ nodes by one dying root.

Figure. 1 shows the initial state of a tree consisting of a single root and illustrates four of the possible states which may be produced by the dying root. The probabilities of producing $0,1,2,3, \ldots$ living nodes are denoted by $f_{0}, f_{1}, f_{2}, f_{3}, \ldots$.

Figure. 2 illustrates one of the realizations of a random tree at the moment $t>0$. In Fig. 2 there are three white circles denoting living nodes which are capable of further evolution, and a large number of black circles denoting dead nodes, which are unable to produce new living nodes. It is to mention that each offspring is connected by a line to its dead precursor. If a dead node has $k$ outgoing lines it is called node of $k$ th out-degree.

In order to simplify our consideration we will assume that the distribution function of the lifetime $\tau$ of living nodes is exponential, i.e.,

$$
d T(t)=e^{-Q t} Q d t
$$

where $1 / Q$ is the expectation value of the lifetime. In this case the evolution becomes a Markovian process. It is assumed that the probabilities

$$
\mathcal{P}\{\nu=k\}=f_{k}, \quad k=0,1,2, \ldots
$$

are the same for all living nodes. By introducing the generating function

$$
q(z)=\sum_{k=0}^{\infty} f_{k} z^{k}
$$

we can define the factorial moments of $\nu$ in the following way:

$$
q_{r}=\left[\frac{d^{r} q(z)}{d z^{r}}\right]_{z=1} \quad r=1,2, \ldots .
$$




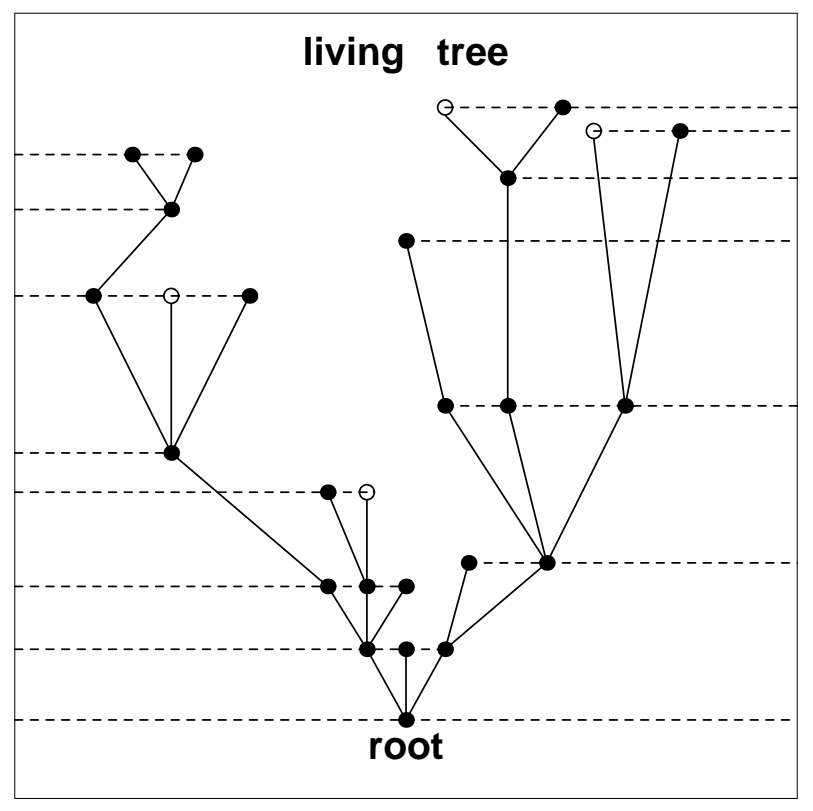

FIG. 2: One of the realizations of a random tree at the time moment $t>0$. The nodes denoted by white circles are capable of further evolution, while those denoted by black cannot evolve and further the horizontal dashed lines indicate the time instances at which nodes were produced.

For the expectation value and the variance of $\nu$ we have

$$
\mathbf{E}\{\nu\}=q_{1} \quad \text { and } \quad \mathbf{D}^{2}\{\nu\}=q_{2}+q_{1}-q_{1}^{2} .
$$

For the sake of later use we cite the relation

$$
\mathbf{E}\left\{(\nu-1)^{2}\right\}=\mathbf{D}^{2}\{\nu\}+\left(1-q_{1}\right)^{2} .
$$

In many cases we do not have to know the exact form of probabilities $f_{k}, k \in \mathcal{Z}$, it is enough to know only the first and second factorial moments of $\nu$. However, if we wish to obtain results which are, in some sense, exact then we will assume that $f_{k}=0, \forall k>2$, i.e. we will use a quadratic generating function

$$
q(z)=f_{0}+f_{1} z+f_{2} z^{2}=1+q_{1}(z-1)+\frac{1}{2} q_{2}(z-1)^{2} .
$$

It seems to be useful to cite the following trivial relations:

$$
f_{0}=1-q_{1}+\frac{1}{2} q_{2}, \quad f_{1}=q_{1}-q_{2}, \quad f_{2}=\frac{1}{2} q_{2},
$$

which follow from the equations $f_{0}+f_{1}+f_{2}=1, \quad f_{1}+2 f_{2}=q_{1}$, and $2 f_{2}=q_{2}$. Since $f_{0}, f_{1}$, and $f_{2}$ are non-negative real numbers smaller than 1 , and their sum is equal to 1 , the possible values of $q_{1}$ and $q_{2}$ are restricted.

As a source of simplification, sometimes we use the geometric distribution

$$
\mathcal{P}\{\nu=k\}=\frac{1}{1+q_{1}}\left(\frac{q_{1}}{1+q_{1}}\right)^{k},
$$

which is defined by the single parameter $q_{1}$ only. The generating function is expressed by

$$
q_{g}(z)=\frac{1}{1+(1-z) q_{1}}
$$

and we find immediately that $\mathbf{E}\{\nu\}=q_{1}$ and $\mathbf{D}^{2}\{\nu\}=q_{1}\left(1+q_{1}\right)$, i.e., $q_{2}=2 q_{1}^{2}$.

In order to characterize the random process of tree evolution we introduce the random functions $\mu_{\ell}(t)$ and $\mu_{d}(t)$ which are the momentary numbers of the living and dead nodes, respectively, at time instant $t \geq 0$. It seems to 
be worthwhile to define the random function $\mu(t)=\mu_{\ell}(t)+\mu_{d}(t)$ which gives the total number of nodes, i.e., the momentary value of the tree size at $t \geq 0$.

The nodes can be sorted according to the number of outgoing lines. Let us denote by $\chi_{\ell}(t, k)$ the number of living, while by $\chi_{d}(t, k)$ the number of dead nodes with $k$ outgoing lines, at the time moment $t$. A node not having any outgoing lines is called an end-node. Obviously, all living nodes are end-nodes, i.e., $\chi_{\ell}(t, 0)=\mu_{\ell}(t)$ and $\chi_{\ell}(t, k)=0$, if $k>0$. Clearly,

$$
\mu_{d}(t)=\sum_{k=0}^{\infty} \chi_{d}(t, k) .
$$

To simplify the notation, we introduce the following random functions:

$$
\chi_{\ell}(t)=\chi_{\ell}(t, 0) \quad \text { and } \quad \chi_{d}(t)=\chi_{d}(t, 0) .
$$

The total number of end-nodes is given by $\chi(t)=\chi_{\ell}(t)+\chi_{d}(t)$. In the next section we shall derive the basic equations for the generating functions of probabilities of the random events $\left\{\mu_{\ell}(t)=n_{\ell}, \mu_{d}(t)=n_{d}\right\}, \quad\{\mu(t)=n\}$ and $\left\{\chi_{\ell}(t)=n_{\ell}, \chi_{d}(t)=n_{d}\right\}, \quad\{\chi(t)=n\}$, provided that at $t=0$ the tree was in the state $\mathcal{S}_{0}$.

\section{DERIVATION OF THE BASIC EQUATIONS}

In this section we would like to deal with equations for the generating functions

$$
g^{(\ell, d)}\left(t, z_{\ell}, z_{d}\right)=\mathbf{E}\left\{z_{\ell}^{\mu_{\ell}(t)} z_{d}^{\mu_{d}(t)}\right\}, \quad \text { and } \quad g(t, z)=\mathbf{E}\left\{z^{\mu(t)}\right\}
$$

as well as for

$$
g_{e}^{(\ell, d)}\left(t, z_{\ell}, z_{d}\right)=\mathbf{E}\left\{z_{\ell}^{\chi_{\ell}(t)} z_{d}^{\chi_{d}(t)}\right\} \quad \text { and } \quad g_{e}(t, z)=\mathbf{E}\left\{z^{\chi(t)}\right\}
$$

It is seen immediately that the generating functions $g^{(\ell)}(t, z)=\mathbf{E}\left\{z^{\mu_{\ell}(t)}\right\}$ and $g^{(d)}(t, z)=\mathbf{E}\left\{z^{\mu_{d}(t)}\right\}$ can be obtained from (8) since

$$
g^{(\ell, d)}\left(t, z_{\ell}=z, z_{d}=1\right)=g^{(\ell)}(t, z), \quad g^{(\ell, d)}\left(t, z_{\ell}=1, z_{d}=z\right)=g^{(d)}(t, z),
$$

and similarly, $g_{e}^{(\ell)}(t, z)$ and $g_{e}^{(d)}(t, z)$ from (9), so it is clear that we need equations only for the generating functions

$$
g^{(\ell, d)}\left(t, z_{\ell}, z_{d}\right), \quad g(t, z) \quad \text { and } \quad g_{e}^{(\ell, d)}\left(t, z_{\ell} z_{d}\right), \quad g_{e}(t, z)
$$

It is evident that these equations belong to the class of equations corresponding to the age-dependent branching processes.

\section{A. Joint distribution of the numbers of living and dead nodes}

We shall define now the probability

$$
\mathcal{P}\left\{\mu_{\ell}(t)=n_{\ell}, \mu_{d}(t)=n_{d} \mid \mathcal{S}_{0}\right\}=p^{(\ell, d)}\left(t, n_{\ell}, n_{d}\right),
$$

where $n_{\ell}$ and $n_{d}$ are non-negative integers. It is clear that $p^{(\ell, d)}\left(t, n_{\ell}, n_{d}\right)$ gives the probability that at the time instant $t \geq 0$ the number of living and that of dead nodes in a randomly evolving tree are equal to $n_{\ell}$ and $n_{d}$, respectively, provided that at $t=0$ the tree was in the state $\mathcal{S}_{0}$. By exploiting the rules of random evolution described in Sec. II and applying the Theorem 7.1 of Ref. [4] it can be shown that

$$
\begin{gathered}
p^{(\ell, d)}\left(t, n_{\ell}, n_{d}\right)=e^{-Q t} \delta_{n_{\ell}, 1} \delta_{n_{d}, 0}+ \\
Q \int_{0}^{t} e^{-Q\left(t-t^{\prime}\right)}\left\{f_{0} \delta_{n_{\ell}, 0} \delta_{n_{d}, 1}+\sum_{k=1}^{\infty} f_{k} V^{(\ell, d)}\left(t^{\prime}, n_{\ell}, n_{d} \mid k\right)\right\} d t
\end{gathered}
$$


where

$$
V^{(\ell, d)}\left(t^{\prime}, n_{\ell}, n_{d} \mid k\right)=\sum_{n_{\ell, 1}+\cdots+n_{\ell, k}=n_{\ell}} \sum_{n_{d, 1}+\cdots+n_{d, k}=n_{d}-1} \prod_{j=1}^{k} p^{(\ell, d)}\left(t^{\prime}, n_{\ell, j}, n_{d, j}\right) .
$$

Introducing the probability generating function

$$
g^{(\ell, d)}\left(t, z_{\ell}, z_{d}\right)=\sum_{n_{\ell}=0}^{\infty} \sum_{n_{d}=0}^{\infty} p^{(\ell, d)}\left(t, n_{\ell}, n_{d}\right) z_{\ell}^{n_{\ell}} z_{d}^{n_{d}},
$$

and taking into account the generating function defined by Eq. (3), we obtain the integral equation in the form

$$
g^{(\ell, d)}\left(t, z_{\ell}, z_{d}\right)=e^{-Q t} z_{\ell}+z_{d} Q \int_{0}^{t} e^{-Q\left(t-t^{\prime}\right)} q\left[g^{(\ell, d)}\left(t^{\prime}, z_{\ell}, z_{d}\right)\right] d t^{\prime},
$$

which according to the Theorem 11.1 of Ref. [4] can be rewritten as a nonlinear differential equation

$$
\frac{\partial g^{(\ell, d)}\left(t, z_{\ell}, z_{d}\right)}{\partial t}=-Q g^{(\ell, d)}\left(t, z_{\ell}, z_{d}\right)+z_{d} Q q\left[g^{(\ell, d)}\left(t, z_{\ell}, z_{d}\right)\right]
$$

with initial condition

$$
\lim _{t \downarrow 0} g^{(\ell, d)}\left(t, z_{\ell}, z_{d}\right)=z_{\ell}
$$

\section{Remarks}

By using the relations $g^{(\ell, d)}\left(t, z_{\ell}=z, z_{d}=1\right)=g^{(\ell)}(t, z), \quad g^{(\ell, d)}\left(t, z_{\ell}=1, z_{d}=z\right)=g^{(d)}(t, z)$ from (12) we obtain the following two equations:

$$
\begin{aligned}
& g^{(\ell)}(t, z)=e^{-Q t} z+Q \int_{0}^{t} e^{-Q\left(t-t^{\prime}\right)} q\left[g^{(\ell)}\left(t^{\prime}, z\right)\right] d t^{\prime}, \\
& g^{(d)}(t, z)=e^{-Q t}+z Q \int_{0}^{t} e^{-Q\left(t-t^{\prime}\right)} q\left[g^{(d)}\left(t^{\prime}, z\right)\right] d t^{\prime} .
\end{aligned}
$$

It is an elementary task to prove that the integral equation (14) is equivalent to the differential equation

$$
\frac{\partial g^{(\ell)}(t, z)}{\partial t}=-Q g^{(\ell)}(t, z)+Q q\left[g^{(\ell)}(t, z)\right]
$$

with the initial condition $\lim _{t \downarrow 0} g^{(\ell)}(t, z)=z$, while Eq.(15) to

$$
\frac{\partial g^{(d)}(t, z)}{\partial t}=-Q g^{(d)}(t, z)+z Q q\left[g^{(d)}(t, z)\right]
$$

with the initial condition $\lim _{t \downarrow 0} g^{(d)}(t, z)=1$.

Finally, it can be easily shown that $g(t, z)=\mathbf{E}\left\{z^{\mu(t)}\right\}$ satisfies the integral equation

$$
g(t, z)=e^{-Q t} z+z Q \int_{0}^{t} e^{-Q\left(t-t^{\prime}\right)} q\left[g\left(t^{\prime}, z\right)\right] d t^{\prime} .
$$

For the sake of completeness we derive from Eq. (18) the equivalent differential equation

$$
\frac{\partial g(t, z)}{\partial t}=-Q g(t, z),+Q z q[g(t, z)]
$$

the initial condition of which is $\lim _{t \downarrow 0} g(t, z)=z$.

It can be shown [15] that each of the equations for the generating functions has just one regular solution. 


\section{B. Joint distribution of the numbers of living and dead end-nodes}

Now we would like to determine the joint distribution of $\chi_{\ell}(t)$ and $\chi_{d}(t)$, i.e., the probability

$$
\mathcal{P}\left\{\chi_{\ell}(t)=n_{\ell}, \chi_{d}(t)=n_{d} \mid \mathcal{S}_{0}\right\}=p_{e}^{(\ell, d)}\left(t, n_{\ell}, n_{d}\right) .
$$

It is evident that

$$
\begin{gathered}
p_{e}^{(\ell, d)}\left(t, n_{\ell}, n_{d}\right)=e^{-Q t} \delta_{n_{\ell}, 1} \delta_{n_{d}, 0}+ \\
+Q \int_{0}^{t} e^{-Q\left(t-t^{\prime}\right)}\left[f_{0} \delta_{n_{\ell}, 0} \delta_{n_{d}, 1}+\sum_{k=1}^{\infty} f_{k} R_{e}^{(\ell, d)}\left(t^{\prime}, n_{\ell}, n_{d} \mid k\right)\right] d t^{\prime}
\end{gathered}
$$

where

$$
R_{e}^{(\ell, d)}\left(t^{\prime}, n_{\ell}, n_{d} \mid k\right)=\sum_{n_{\ell, 1}+\cdots+n_{\ell, k}=n_{\ell}} \sum_{n_{d, 1}+\cdots+n_{d, k}=n_{d}} \prod_{j=1}^{k} p_{e}^{(\ell, d)}\left(t^{\prime}, n_{\ell, j}, n_{d, j}\right) .
$$

Simple calculations show that the generating function

$$
g_{e}^{(\ell, d)}\left(t, z_{\ell}, z_{d}\right)=\sum_{n_{\ell}=0}^{\infty} \sum_{n_{d}=0}^{\infty} p_{e}^{(\ell, d)}\left(t, n_{\ell}, n_{d}\right) z_{\ell}^{n_{\ell}} z_{d}^{n_{d}}
$$

satisfies the equation

$$
g_{e}^{(\ell, d)}\left(t, z_{\ell}, z_{d}\right)=e^{-Q t} z_{\ell}-f_{0}\left(1-z_{d}\right)\left(1-e^{-Q t}\right)+Q \int_{0}^{t} e^{-Q\left(t-t^{\prime}\right)} q\left[g_{e}^{(\ell, d)}\left(t, z_{\ell}, z_{d}\right)\right] d t^{\prime},
$$

which will be used for the determination of the correlation between the random variables $\chi_{\ell}(t)$ and $\chi_{d}(t)$. For the sake of brevity the corresponding differential equation is omitted.

\section{Remark}

There is no need to write down the equation for generating function of $\chi_{\ell}(t)$, since $g_{e}^{(\ell)}(t, z)=g^{\ell}(t)$ which is the solution of Eq. (14). The equation for the generating function $g_{e}^{(d)}(t, z)$, however, one can obtain from (19) by substitutions $z_{\ell}=1$ and $z_{d}=z$, and so one has

$$
g_{e}^{(d)}(t, z)=e^{-Q t}-(1-z) f_{0}\left(1-e^{-Q t}\right)+Q \int_{0}^{t} e^{-Q\left(t-t^{\prime}\right)} q\left[g_{e}^{(d)}\left(t^{\prime}, z\right)\right] d t^{\prime} .
$$

In order to gain an insight into the interplay between the living and dead end-nodes it seems to be useful to calculate the probability distribution of the random function $\chi(t)=\chi_{\ell}(t)+\chi_{d}(t)$. The equation for the generating function is easily obtained as

$$
g_{e}(t, z)=\sum_{n=0}^{\infty} \mathcal{P}\left\{\chi(t)=n \mid \mathcal{S}_{0}\right\} z^{n}=\sum_{n=0}^{\infty} p_{e}(t, n) z^{n},
$$

where $p_{e}(t, n)$ is the probability that the number of all end-nodes at $t \geq 0$ is equal to $n$ provided that at $t=0$ the tree was in the state $\mathcal{S}_{0}$. Applying the usual argumentations one has

$$
g_{e}(t, z)=e^{-Q t} z-(1-z) f_{0}\left(1-e^{-Q t}\right)+Q \int_{0}^{t} e^{-Q\left(t-t^{\prime}\right)} q\left[g_{e}\left(t^{\prime}, z\right)\right] d t^{\prime} .
$$




\section{AVERAGE CHARACTERISTICS}

\section{A. Correlations between the numbers of living and dead nodes}

The study of how the random functions $\mu_{\ell}(t)$ and $\mu_{d}(t)$ are related involves the analysis of correlation between them. First we should calculate the covariance function

$$
\operatorname{Cov}\left\{\mu_{\ell}(t) \mu_{d}(t)\right\}=\mathbf{E}\left\{\mu_{\ell}(t) \mu_{d}(t)\right\}-\mathbf{E}\left\{\mu_{\ell}(t)\right\} \mathbf{E}\left\{\mu_{d}(t)\right\},
$$

and then the correlation function

$$
C^{(\ell, d)}\left(t, q_{1}, q_{2}\right)=\frac{\operatorname{Cov}\left\{\mu_{\ell}(t) \mu_{d}(t)\right\}}{\mathbf{D}\left\{\mu_{\ell}(t)\right\} \mathbf{D}\left\{\mu_{d}(t)\right\}} .
$$

As is apparent, we must determine the expectations and variances of $\mu_{\ell}(t)$ and $\mu_{d}(t)$. Taking into account the relationships

$$
\mathbf{E}\left\{\mu_{\ell}(t)\right\}=\left(\frac{\partial g^{(\ell)}(t, z)}{\partial z}\right)_{z=1}=m_{1}^{(\ell)}(t)
$$

and

$$
\mathbf{E}\left\{\mu_{d}(t)\right\}=\left(\frac{\partial g^{(d)}(t, z)}{\partial z}\right)_{z=1}=m_{1}^{(d)}(t)
$$

after simple algebra we find

$$
m_{1}^{(\ell)}(t)=e^{-\alpha t} \quad \text { and } \quad m_{1}^{(d)}(t)= \begin{cases}\frac{1}{1-q_{1}}\left[1-e^{-\alpha t}\right], & \text { if } q_{1} \neq 1, \\ Q t, & \text { if } q_{1}=1,\end{cases}
$$

where $\alpha=\left(1-q_{1}\right) Q$. It is here to mention that the tree evolution is called subcritical, if $q_{1}<1$, critical, if $q_{1}=1$, and supercritical, if $q_{1}>1$.

The formulas

$$
\mathbf{D}^{2}\left\{\mu_{\ell}(t)\right\}=m_{2}^{(\ell)}(t)+m_{1}^{(\ell)}(t)\left[1-m_{1}^{(\ell)}(t)\right]
$$

and

$$
\mathbf{D}^{2}\left\{\mu_{d}(t)\right\}=m_{2}^{(d)}(t)+m_{1}^{(d)}(t)\left[1-m_{1}^{(d)}(t)\right]
$$

show that we must determine the second factorial moments

$$
m_{2}^{(\ell)}(t)=\left(\frac{\partial^{2} g^{(\ell)}(t, z)}{\partial z^{2}}\right)_{z=1} \quad \text { and } \quad m_{2}^{(d)}(t)=\left(\frac{\partial^{2} g^{(d)}(t, z)}{\partial z^{2}}\right)_{z=1} .
$$

After elementary calculations we find that

$$
m_{2}^{(\ell)}(t)= \begin{cases}\frac{q_{2}}{1-q_{1}}\left[1-e^{-\alpha t}\right] e^{-\alpha t}, & \text { if } q_{1} \neq 1 \\ q_{2} Q t, & \text { if } q_{1}=1\end{cases}
$$

and

$$
m_{2}^{(d)}(t)= \begin{cases}a_{1}^{(d)}\left[1-e^{-\alpha t}\right]+a_{2}^{(d)} Q t e^{-\alpha t}+a_{3}^{(d)} e^{-\alpha t}\left[1-e^{-\alpha t}\right], & \text { if } q_{1} \neq 1 \\ (Q t)^{2}+\frac{1}{3} q_{2}(Q t)^{3}, & \text { if } q_{1}=1\end{cases}
$$

where

$$
a_{1}^{(d)}=\frac{1}{\left(1-q_{1}\right)^{2}}\left(2 q_{1}+\frac{q_{2}}{1-q_{1}}\right), \quad a_{2}^{(d)}=-\frac{2}{1-q_{1}}\left(q_{1}+\frac{q_{2}}{1-q_{1}}\right)
$$


and

$$
a_{3}^{(d)}=\frac{q_{2}}{\left(1-q_{1}\right)^{3}} .
$$

By substituting the first and second factorial moments of Eq. (25) for formulas (24) and (27), we obtain that

$$
\mathbf{D}^{2}\left\{\mu_{\ell}(t)\right\}= \begin{cases}\frac{\mathbf{E}\left\{(\nu-1)^{2}\right\}}{1-q_{1}}\left[1-e^{-\alpha t}\right] e^{-\alpha t}, & \text { if } q_{1} \neq 1, \\ \mathbf{D}^{2}\{\nu\} Q t, & \text { if } q_{1}=1,\end{cases}
$$

where $\mathbf{E}\left\{(\nu-1)^{2}\right\}=\left(1-q_{1}\right)^{2}+\mathbf{D}^{2}\{\nu\} .^{2}$ Applying similar substitution in (26), if $q_{1} \neq 1$, then we arrive at

$$
\mathbf{D}^{2}\left\{\mu_{d}(t)\right\}=v_{1}^{(d)}\left[1-e^{-\alpha t}\right]+v_{2}^{(d)} Q t e^{-\alpha t}+v_{3}^{(d)} e^{-\alpha t}\left[1-e^{-\alpha t}\right],
$$

where

$$
v_{1}^{(d)}=\frac{\mathbf{D}^{2}\{\nu\}}{\left(1-q_{1}\right)^{3}}, \quad v_{2}^{(d)}=-2 \frac{\mathbf{D}^{2}\{\nu\}}{\left(1-q_{1}\right)^{2}}, \quad v_{3}^{(d)}=\frac{\mathbf{E}\left\{(\nu-1)^{2}\right\}}{\left(1-q_{1}\right)^{3}} .
$$

If $q_{1}=1$, i.e., the evolution is critical, then

$$
\mathbf{D}^{2}\left\{\mu_{d}(t)\right\}=Q t+\frac{1}{3} q_{2}(Q t)^{3} .
$$

One can see that the limit values of the variance of the number of dead nodes are given by the formula

$$
\lim _{t \rightarrow \infty} \mathbf{D}^{2}\left\{\mu_{d}(t)\right\}= \begin{cases}\frac{\mathbf{D}^{2}\{\nu\}}{\left(1-q_{1}\right)^{3}}, & \text { if } q_{1}<1 \\ \infty, & \text { if } q_{1} \geq 1\end{cases}
$$

In order to calculate the covariance function we should solve also the equation determining the mixed moment

$$
\mathbf{E}\left\{\mu_{\ell}(t) \mu_{d}(t)\right\}=\left[\frac{\partial^{2} g^{(\ell, d)}\left(t, z_{\ell}, z_{d}\right)}{\partial z_{\ell} \partial z_{d}}\right]_{z_{\ell}=z_{d}=1}=m_{1,1}^{(\ell, d)}(t) .
$$

The equation can be written in the form

$$
\begin{gathered}
m_{1,1}^{(\ell, d)}(t)=Q q_{1} \int_{0}^{t} e^{-Q\left(t-t^{\prime}\right)} m_{1,1}^{(\ell, d)}\left(t^{\prime}\right) d t^{\prime}+ \\
Q \int_{0}^{t} e^{-Q\left(t-t^{\prime}\right)}\left[q_{1} m_{1}^{(\ell)}\left(t^{\prime}\right)+q_{2} m_{1}^{(\ell)}\left(t^{\prime}\right) m_{1}^{(d)}\left(t^{\prime}\right)\right] d t^{\prime} .
\end{gathered}
$$

By using Eq. (24) for $m_{1}^{(\ell)}(t)$ and $m_{1}^{(d)}(t)$, and introducing the notation $\alpha=\left(1-q_{1}\right) Q$, it can be proved that

$$
m_{1,1}^{(\ell, d)}(t)= \begin{cases}\frac{\mathbf{D}^{2}\{\nu\}}{1-q_{1}} Q t e^{-\alpha t}-q_{2} \frac{1}{\left(1-q_{1}\right)^{2}} e^{-\alpha t}\left(1-e^{-\alpha t}\right), & \text { if } q_{1} \neq 1, \\ Q t+\frac{1}{2} \mathbf{D}^{2}\{\nu\}(Q t)^{2}, & \text { if } q_{1}=1\end{cases}
$$

is the unique solution of the integral equation (31). After a brief calculation we have

$$
\operatorname{Cov}\left\{\mu_{\ell}(t) \mu_{d}(t)\right\}= \begin{cases}\frac{\mathbf{D}^{2}\{\nu\}}{1-q_{1}} Q t e^{-\alpha t}-\left[1+\frac{\mathbf{D}^{2}\{\nu\}}{\left(1-q_{1}\right)^{2}}\right] e^{-\alpha t}\left(1-e^{-\alpha t}\right), & \text { if } q_{1} \neq 1, \\ \frac{1}{2} \mathbf{D}^{2}\{\nu\}(Q t)^{2}, & \text { if } q_{1}=1\end{cases}
$$

\footnotetext{
${ }^{2}$ In the case of subcritical evolution the variance has a maximum at $t_{\max }=\log 2 / \alpha$ which, as seen, is independent of the form of the distribution of $\nu$.
} 


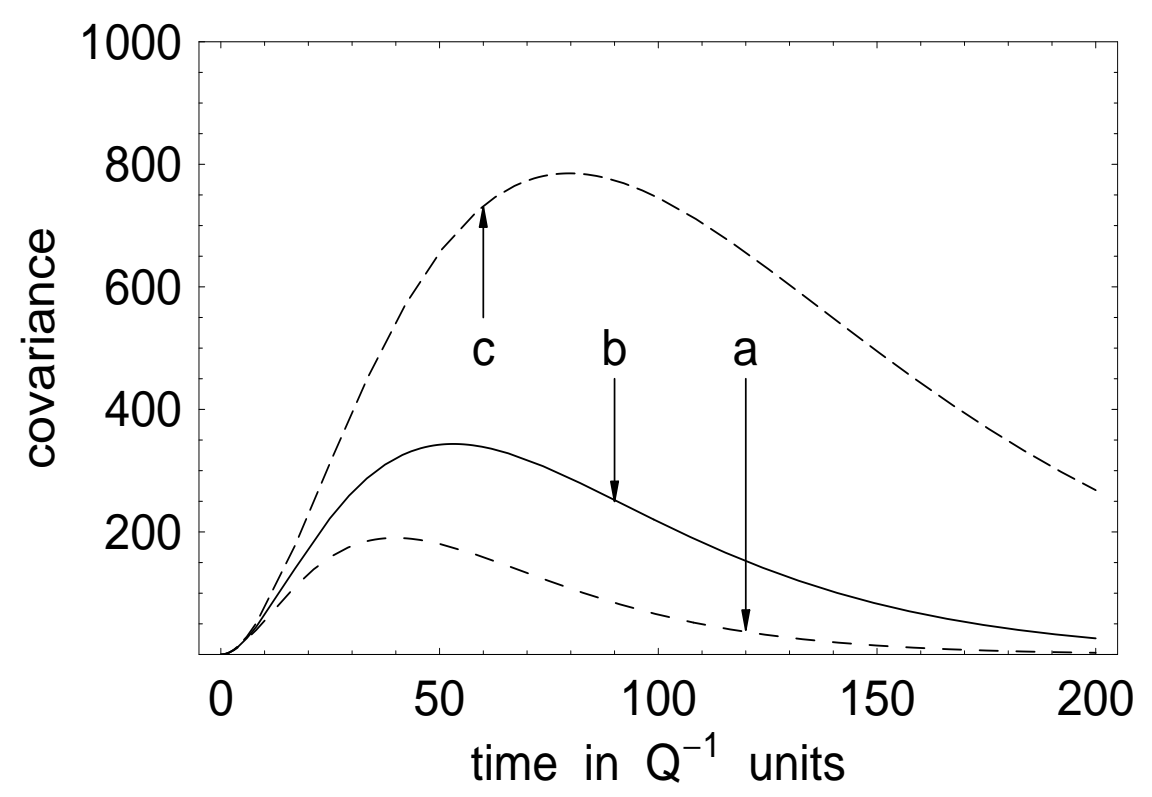

FIG. 3: Three curves illustrating the time dependence of the covariance $\operatorname{Cov}\left\{\mu_{\ell}(t) \mu_{d}(t)\right\}$ in the case of subcritical random evolution with the assumption that $\nu$ is of geometric distribution. The curves $\mathbf{a}, \mathbf{b}$, and $\mathbf{c}$ correspond to the values of $q_{1}=0.96,0.97$ and 0.98 , respectively.

The dependence of the covariance function $\operatorname{Cov}\left\{\mu_{\ell}(t) \mu_{d}(t)\right\}$ on the time parameter $Q t$ at three different values of $q_{1}$ has been calculated by assuming that $\nu$ is of geometric distribution. The three curves $\mathbf{a}, \mathbf{b}$, and $\mathbf{c}$ corresponding to the values $q_{1}=0.96,0.97$, and 0.98 , respectively, are plotted in Fig. 3. One may observe that each of the covariance curves vs time has a well defined maximum. The time parameter which belongs to the maximum of the covariance function depends rather sensitively on $q_{1}$.

Now, we would like to return to the study of the correlation function defined by (23). It is evident that the numbers of living and dead nodes are correlated, but it is worthwhile to note that the time dependence of the correlation function $C^{(\ell, d)}\left(t, q_{1}, q_{2}\right)$ has some specific features, which follow from properties of the covariance function and the variances of $\mu_{\ell}(t)$ and $\mu_{d}(t)$. For example, one can show that in the case of subcritical evolution the correlation function, after a sharp increase, converges to zero when $Q t \rightarrow \infty$, while in the case of supercritical evolution it rapidly

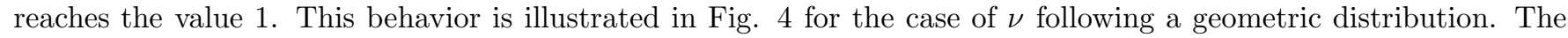
curves $\mathbf{a}, \mathbf{b}$, and $\mathbf{c}$ belong to values $0.95,1$, and 1.05 of $q_{1}$, respectively. It seems to be interesting to present some curves reflecting the dependence of $C^{(\ell, d)}\left(t, q_{1}, q_{2}\right)$ on $q_{1}$ at different time instants. In Fig. 5 we can see that the transition of the correlation function from 0 to 1 becomes sharper and sharper as $Q t$ increases. The curves $\mathbf{a}$ and $\mathbf{b}$ belong to the time parameters $Q t=100$ and $Q t=500$, respectively, while curve c shows how the correlation function depends on $q_{1}$ at $Q t=\infty$.

The most surprising result can be obtained when the random evolution is critical, i.e., $q_{1}=1$. Then we have

$$
C^{(\ell, d)}\left(t, 1, q_{2}\right)=\frac{\sqrt{3}}{2}\left[1+\frac{3}{\mathbf{D}^{2}\{\nu\}(Q t)^{2}}\right]^{-1 / 2}
$$

which shows that the limit value of the correlation

$$
\lim _{Q t \rightarrow \infty} C^{(\ell, d)}\left(t, 1, q_{2}\right)=\frac{\sqrt{3}}{2}
$$

is independent of the distributions of $\nu$ and $\tau$. This very important limit law expresses the fact that the relation between the numbers of living and dead nodes near the critical state, and at time moments sufficiently far from the beginning of the process, becomes almost independent of rules controlling the evolution. This independence can be attributed to the appearance of the universal properties of the evolution. 


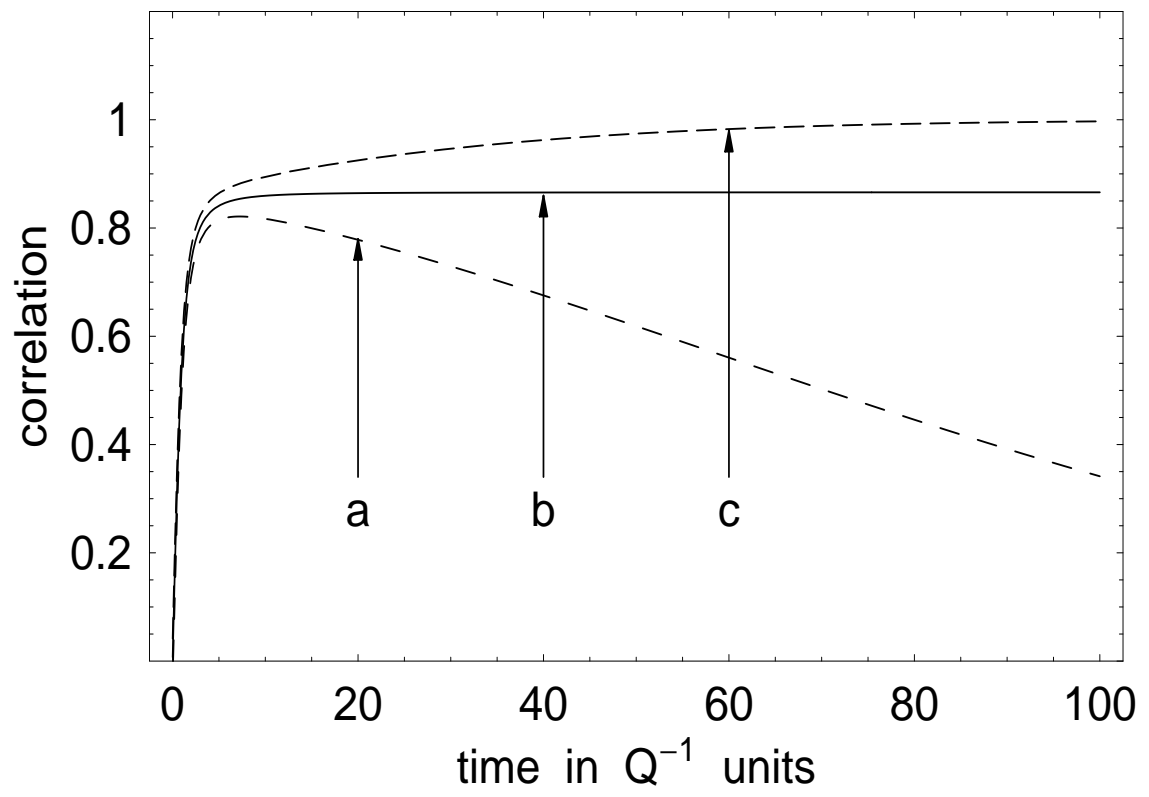

FIG. 4: Time dependence of the correlation between the numbers living and dead nodes if $\nu$ is of geometric distribution. The curves a, $\mathbf{b}$, and $\mathbf{c}$ belong to $q_{1}$ values $0.95,1$, and 1.05 , respectively.

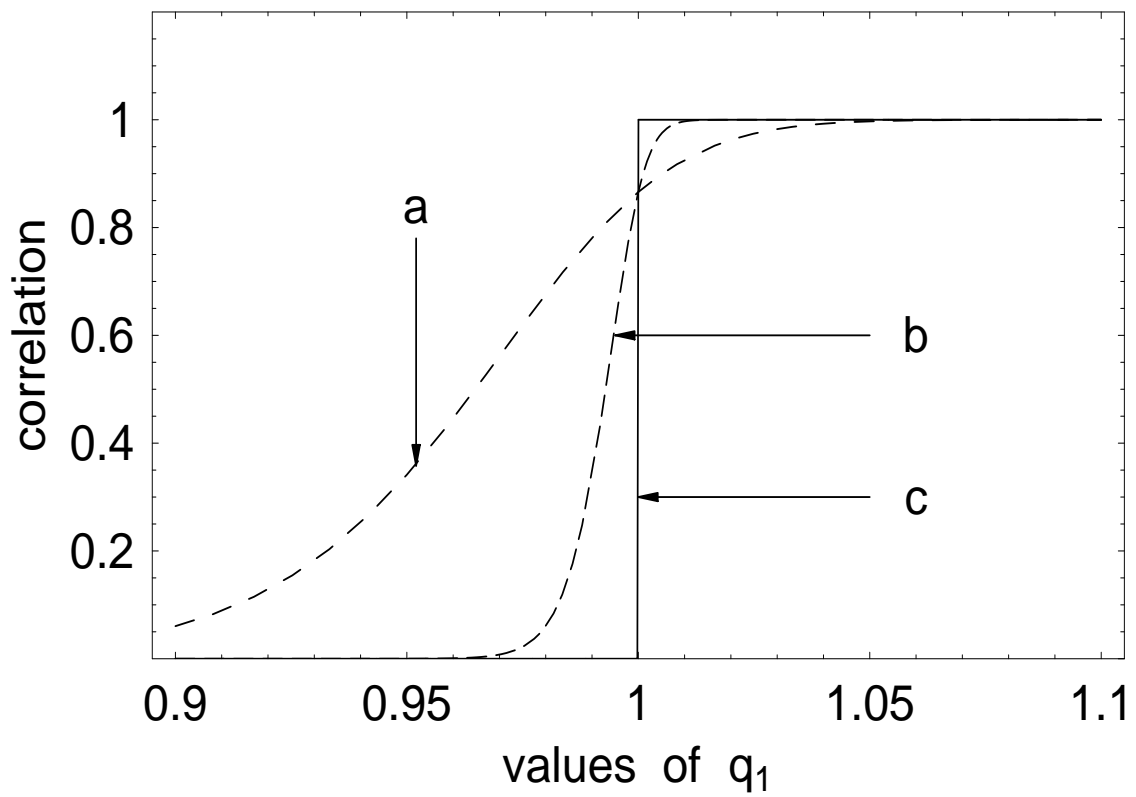

FIG. 5: Dependence of the correlation between the numbers of living and dead nodes on $q_{1}$ at time instants $Q t=100,(\mathbf{a}), Q t=500,(\mathbf{b})$ and $Q t=\infty,(\mathbf{c})$.

\section{B. Correlations between the numbers of living and dead end-nodes}

The dynamics of the random tree evolution is determined by the end-nodes. It is evident that living end-nodes are responsible for the development of a tree, while dead end-nodes represent points where the tree is not capable of any variation. In order to have an insight into the dynamics of the tree evolution it is useful to study the correlation function of $\chi_{\ell}(t)$ and $\chi_{d}(t)$. First we calculate the covariance function

$$
\operatorname{Cov}\left\{\chi_{\ell}(t) \chi_{d}(t)\right\}=\mathbf{E}\left\{\chi_{\ell}(t) \chi_{d}(t)\right\}-\mathbf{E}\left\{\chi_{\ell}(t)\right\} \mathbf{E}\left\{\chi_{d}(t)\right\},
$$


and then the correlation function

$$
C_{e}^{(\ell, d)}\left(t, q_{1}, q_{2}\right)=\frac{\operatorname{Cov}\left\{\chi_{\ell}(t) \chi_{d}(t)\right\}}{\mathbf{D}\left\{\chi_{\ell}(t)\right\} \mathbf{D}\left\{\chi_{d}(t)\right\}} .
$$

Since $\chi_{\ell}(t)=\mu_{\ell}(t)$, we have to deal only with the determination of the variance $\mathbf{D}^{2}\left\{\chi_{d}\right\}$. Omitting the details of calculations, if $q_{1} \neq 1$, we have

$$
\begin{gathered}
\mathbf{D}^{2}\left\{\chi_{d}(t)\right\}=\frac{f_{0}^{2}}{\left(1-q_{1}\right)^{2}}\left[\left(\frac{q_{2}}{1-q_{1}}+\frac{1-q_{1}}{f_{0}}-1\right)\left(1-e^{-\alpha t}\right)-2 q_{2} Q t e^{-\alpha t}+\right. \\
\left.+\left(\frac{q_{2}}{1-q_{1}}+1\right)\left(1-e^{-\alpha t}\right) e^{-\alpha t}\right],
\end{gathered}
$$

while, if $q_{1}=1$, we obtain the following simple formula:

$$
\mathbf{D}^{2}\left\{\chi_{d}(t)\right\}=f_{0} Q t\left(1+\frac{1}{3} q_{2}(q t)^{2}\right) .
$$

It is worthwhile to remark that if the tree evolution is subcritical, then and only then does the variance of the number of dead end-nodes converge to a finite limit value given by

$$
\lim _{t \rightarrow \infty} \mathbf{D}^{2}\left\{\chi_{d}(t)\right\}=\frac{f_{0}^{2}}{\left(1-q_{1}\right)^{2}}\left(\frac{q_{2}}{1-q_{1}}+\frac{1-q_{1}}{f_{0}}-1\right) .
$$

As regards the covariance function from the generating function $g_{e}^{(\ell, d)}\left(t, z_{\ell}, z_{d}\right)$ defined by (19) we should derive the second mixed moment

$$
m_{1,1}^{(\ell, d)}(t \mid e)=\left(\frac{\partial^{2} g_{e}^{(\ell, d)}\left(t, z_{\ell}, z_{d}\right)}{\partial z_{\ell} \partial z_{d}}\right)_{z_{\ell}=z_{d}=1}
$$

which is determined by the following integral equation:

$$
m_{1,1}^{(\ell, d)}(t \mid e)=q_{1} Q \int_{0}^{t} e^{-Q\left(t-t^{\prime}\right)} m_{1,1}^{(\ell, d)}\left(t^{\prime} \mid e\right) d t^{\prime}+q_{2} Q \int_{0}^{t} e^{-Q\left(t-t^{\prime}\right)} m_{1}^{(\ell)}\left(t^{\prime} \mid e\right) m_{1}^{(d)}\left(t^{\prime} \mid e\right) d t^{\prime} .
$$

Since

$$
m_{1}^{(\ell)}(t \mid e)=m_{1}^{(\ell)}(t)=e^{-\alpha t} \quad \text { and } \quad m_{1}^{(d)}(t)=f_{0} \frac{1-e^{-\alpha t}}{1-q_{1}}
$$

we obtain the solution in the form:

$$
\operatorname{Cov}\left\{\chi_{\ell}(t) \chi_{d}(t)\right\}= \begin{cases}\frac{f_{0}}{1-q_{1}}\left[q_{2} Q t-\left(\frac{q_{2}}{1-q_{1}}+1\right)\left(1-e^{-\alpha t}\right)\right] e^{-\alpha t}, & \text { if } q_{1} \neq 1 \\ f_{0} Q t\left(\frac{1}{2} q_{2} Q t-1\right), & \text { if } q_{1}=1\end{cases}
$$

As is to be seen in Fig. [6] the covariance of the numbers of living and dead end-nodes changes its sign at the very beginning of the evolution. The negative covariance just after the start is a consequence of the simple fact that the root of the tree can be but a living end-node at the time instant $t=0$. The calculations refer to the case when $\nu$ is of geometric distribution; this however, does not affect the generality of the conclusion.

For the sake of completeness we calculated the time dependence of the correlation function of the numbers of living and dead end-nodes at values of $q_{1}=0.95,1,1.05$ assuming again that $\nu$ is of geometric distribution. As expected, Fig. 7 shows convincingly the change realizing in the character of the correlation near the beginning of the evolution. There is no need to write down the lengthy expression of the correlation function but it seems to be useful to present it when the tree evolution is critical. In this case one obtains

$$
C_{e}^{(\ell, d)}(t)=\frac{1}{2} \sqrt{\frac{3 f_{0}}{q_{2}}} \frac{q_{2} Q t-2}{\sqrt{3+q_{2}(Q t)^{2}}} .
$$

It is interesting to note that the correlation in critical evolution reaches its asymptotic value $\sqrt{3 f_{0}} / 2$ very soon. (If $f_{0}=0.5$, then $C_{e}^{(\ell, d)}(\infty) \approx 0.612$ and this is what is seen in Fig. (7) 

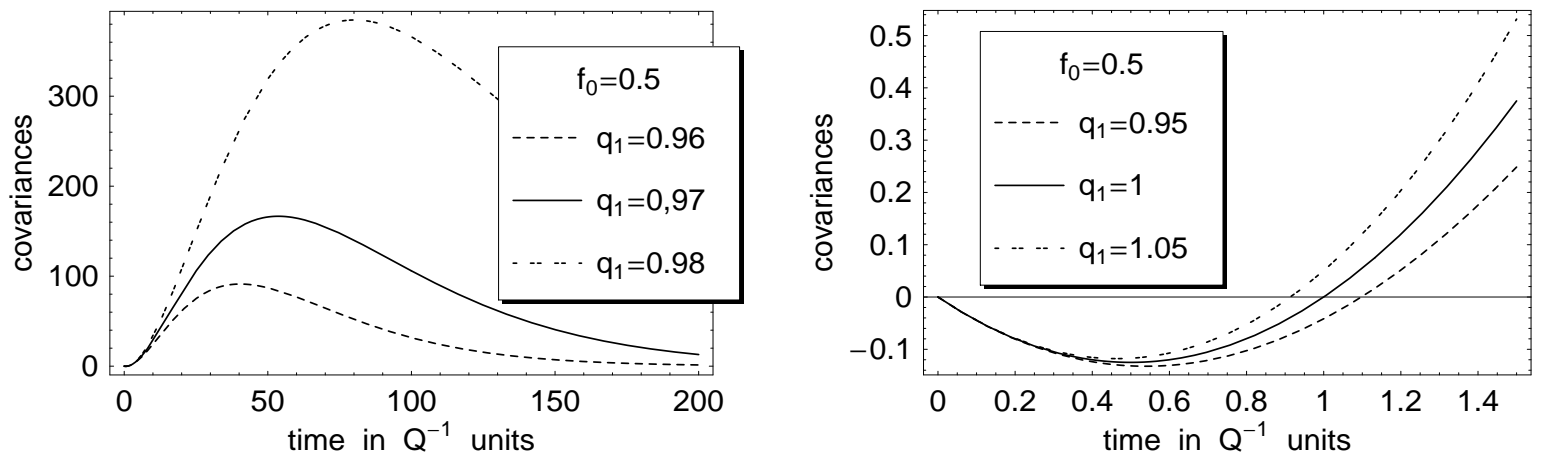

FIG. 6: Dependence of the covariance of the numbers of living and dead end-nodes on $Q t$ at different values of $q_{1}$.

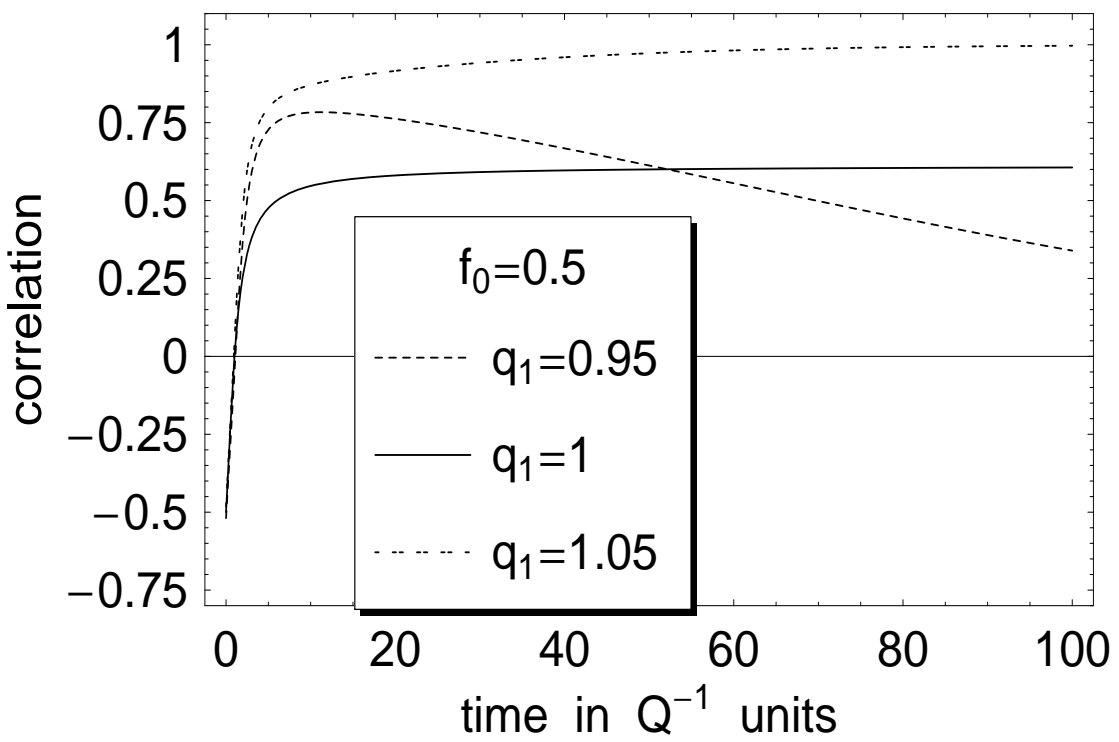

FIG. 7: Dependence of the correlation between the numbers of living and dead end-nodes on $Q t$ at different values of $q_{1}$.

\section{SURVIVAL PROBABILITY}

\section{A. General considerations}

It is obvious that the evolution of a random tree will stop at the time instant $\theta$ which satisfies the equation $\mu_{\ell}(\theta)=0$ with probability 1 . The random variable $\theta$ is called the lifetime of the tree. In order to determine its distribution function

$$
\mathcal{P}\left\{\theta \leq t \mid \mathcal{S}_{0}\right\}=L(t),
$$

one must recognize that the probability $\mathcal{P}\left\{\mu_{\ell}(t)=0 \mid \mathcal{S}_{0}\right\}=p^{(\ell)}(t, 0)$ to find zero living node at the time moment $t \geq 0$ in a tree is the same as the probability that the lifetime $\theta$ of that tree is not larger than $t \geq 0$, therefore, one can write $\mathcal{P}\left\{\theta \leq t \mid \mathcal{S}_{0}\right\}=\mathcal{P}\left\{\mu_{\ell}(t)=0 \mid \mathcal{S}_{0}\right\}$, i.e.,

$$
L(t)=p^{(\ell)}(t, 0)=\lim _{z \downarrow 0} g^{(\ell)}(t, z) .
$$

It is clear that if $0<t_{1} \leq t_{2}$ then $L\left(t_{1}\right) \leq L\left(t_{2}\right)$, i.e., $L(t)$ is a nondecreasing function of its argument, hence the limit relation

$$
\max _{0<t \leq \infty} L(t)=\lim _{t \rightarrow \infty} L(t)=L_{\infty} \leq 1
$$


must hold. We will call the quantity $L_{\infty}$ extinction probability, and will now prove the following statement: if $q_{1} \leq 1$, i.e., the random evolution is not supercritical, then $L_{\infty}=1$, while if $q_{1}>1$, i.e., the evolution is supercritical, then $L_{\infty}$ is equal to the non-negative, single root of the function $\psi(y)=q(y)-y, y \in[0,1]$. (It is evident that $\psi(1)=0$.)

For the proof we exploit the fundamental property of the generating function $g^{(\ell)}(t, z)$ which is expressed by the equation $g^{(\ell)}(t+u, z)=g^{(\ell)}\left[t, g^{(\ell)}(u, z)\right]$. Applying the relation 46] we have $L(t+u)=g^{(\ell)}[t, L(u)]$ and since

$$
\lim _{u \rightarrow \infty} L(t+u)=\lim _{u \rightarrow \infty} L(u)=L_{\infty}
$$

we can write for every $t \geq 0$ that $L_{\infty}=g^{(\ell)}\left(t, L_{\infty}\right)$. Plugging $g^{(\ell)}\left(t, L_{\infty}\right)$ into the equation

$$
\frac{\partial g^{(\ell)}(t, z)}{\partial}=Q q\left[g^{(\ell)}(t, z)\right]-Q g^{(\ell)}(t, z),
$$

we obtain

$$
q\left(L_{\infty}\right)-L_{\infty}=0
$$

Considering that $q(y)$ is a probability generating function, i.e., $\lim _{y \uparrow 1} q(y)=1$, then according to a well-known theorem of generating functions, it is clear that if $q_{1}>1$ then Eq. (48) besides the trivial fixed-point 1 must have also another, smaller than 1, non-negative fixed-point $L_{\infty}$, and this is what we wanted to prove.

Now, we would like to define the survival probability. Obviously,

$$
S(x)=1-L(x)
$$

is the probability to find the tree at the time instant $x=Q t$ in a living state, and therefore, $S(x)$ can be called the survival probability. By taking into account the properties of $L(x)$ one obtains

$$
\lim _{Q t \rightarrow \infty} S(x)= \begin{cases}0, & \text { if } q_{1} \leq 1 \\ S_{\infty}=1-L_{\infty}, & \text { if } q_{1}>1\end{cases}
$$

Clearly, from Eqs. (47), (45), and (49) one can derive the equation

$$
\frac{d S}{d x}=-q(1-S)-S+1
$$

which has the solution

$$
x(S)=\int_{S(x)}^{1} \frac{d y}{q(1-y)+y-1},
$$

at the initial condition $S(0)=1$.

\section{B. Calculations in the case of a quadratic $q(z)$ function}

In order to demonstrate the characteristic features of the lifetime of random trees we will determine the survival probability in the case when the generating function of the offspring number $\nu$ is a quadratic expression defined by (6). By using Eq. (51) we obtain

$$
\frac{d S}{d x}=-\left(1-q_{1}\right) S-\frac{1}{2} q_{2} S^{2}
$$

and taking into account the initial condition $S(0)=1$ we have the solution in the form

$$
S(x)= \begin{cases}e^{-\left(1-q_{1}\right) x}\left[1+\frac{q_{2}}{2\left(1-q_{1}\right)}\left(1-e^{-\left(1-q_{1}\right) x}\right)\right]^{-1}, & \text { if } q_{1}<1 \\ \frac{2}{2+q_{2} x}, & \text { if } q_{1}=1 \\ 2 \frac{q_{1}-1}{q_{2}}\left[1+\left(1-2 \frac{q_{1}-1}{q_{2}}\right) e^{-\left(q_{1}-1\right) x}\right]^{-1}, & \text { if } q_{1}>1 .\end{cases}
$$




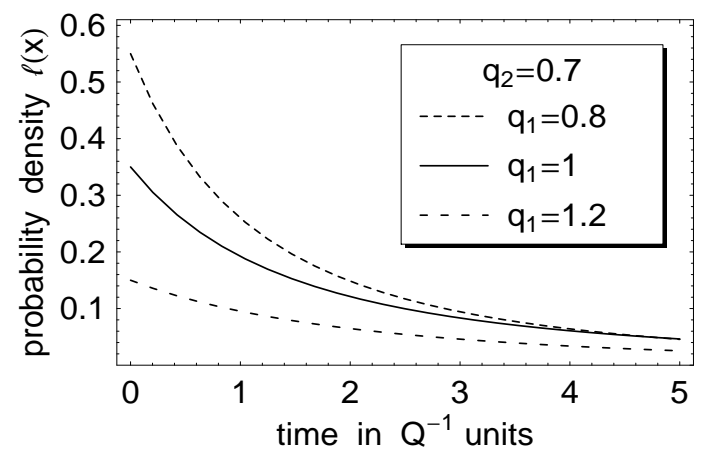

FIG. 8: Dependence of the probability density function of the lifetime on $x=Q t$.

The density function of the lifetime measured in units of $Q^{-1}$ can be calculated by using the relation

$$
\ell(x)=\frac{d L(x)}{d x}=-\frac{d S(x)}{d x} .
$$

It is elementary to show that the density function is decreasing monotonously from $\ell(0)=f_{0}$ to zero. In Fig. 8 one can see the density function curves versus time $x=Q t$ for subcritical, critical, and supercritical trees.

From (54) one can see that

$$
\lim _{x=Q t \rightarrow \infty} S(x)= \begin{cases}0, & \text { if } q_{1} \leq 1, \\ S_{\infty}=2 \frac{q_{1}-1}{q_{2}}, & \text { if } q_{1}>1 .\end{cases}
$$

For more detailed analysis of asymptotic values for $S(x)$ see Ref. 14].

Finally, in this section let us calculate the expectation and the variance of the tree lifetime $\theta$ in units of $Q^{-1}$. For this purpose it seems to be useful to determine the characteristic function of the random variable $Q \theta$. Since the moments $\mathbf{E}\left\{(Q \theta)^{j}\right\}, \quad j=1,2, \ldots$ do not exist if $q_{1} \geq 1$, the calculations are restricted to the case when $q_{1}<1$. One can write

$$
\varphi(\omega)=\mathbf{E}\left\{e^{-\omega Q \theta}\right\}=\int_{0}^{\infty} e^{-\omega x} d L(x)=\int_{0}^{1} e^{-\omega x(y)} d y
$$

where $\omega$ is a complex number with Rew $\geq 0$. Performing the substitution

$$
x(y)=-\frac{1}{1-q_{1}} \ln y \frac{1+2 \frac{1-q_{1}}{q_{2}}}{y+2 \frac{1-q_{1}}{q_{2}}}
$$

one obtains the characteristic function

$$
\varphi(\omega)=(1+\gamma)^{\omega \beta} \int_{0}^{1}\left[\frac{y}{y+\gamma}\right]^{\omega \beta} d y
$$

where

$$
\beta=\left(1-q_{1}\right)^{-1} \quad \text { and } \quad \gamma=2 \frac{1-q_{1}}{q_{2}}, \quad q_{1}<1 .
$$

From the characteristic functions $\varphi(\omega)$ both the expectation and the variance of the lifetime can be easily calculated when the tree evolution is subcritical. For the expectation value one obtains

$$
\mathbf{E}\{Q \theta\}=-\left(\frac{d \varphi(\omega)}{d \omega}\right)_{\omega=0}=\frac{2}{q_{2}} \ln \left(1+\frac{1}{2} \frac{q_{2}}{1-q_{1}}\right) .
$$

If $q_{1} \rightarrow 1$, then the expectation value diverges as $\ln \left(1-q_{1}\right)^{-1}$. 
For the calculation of the variance we need the second moment of $Q \theta$, which can be immediately obtained from the characteristic function. Omitting the details of the calculation we may write

$$
\begin{gathered}
\mathbf{D}^{2}\{Q \theta\}= \\
=-\left(\frac{2}{q_{2}}\right)^{2}\left(1+\frac{1}{2} \frac{q_{2}}{1-q_{1}}\right)\left[\ln \left(1+\frac{1}{2} \frac{q_{2}}{1-q_{1}}\right)\right]^{2}-\frac{4}{q_{2}\left(1-q_{1}\right)} L i_{2}\left(-\frac{1}{2} \frac{q_{2}}{1-q_{1}}\right),
\end{gathered}
$$

where $L i_{2}(u)=\sum_{k=1}^{\infty} u^{k} / k^{2} \quad$ is the so called Jonquière's function. When $q_{1}$ is approaching 1 from below, the fluctuation of the tree lifetime becomes unlimitedly large, and so in the vicinity of the critical evolution the average lifetime loses almost completely its information content.

\section{CONCLUSIONS}

Let us summarize the main results of the paper. By introducing the notions of living and dead nodes, a model of random tree evolution with continuous time parameter has been constructed. The model describes the spreading in time of abstract objects which correspond to nodes. It is to mention that the process of the random tree evolution analyzed in this paper belongs to the family of the age-dependent branching processes.

In order to characterize the evolution process, essentially two basic random functions $\mu_{\ell}(t)$ and $\mu_{d}(t)$ have been used. The first one is the momentary number of living nodes, while the second one is that of dead nodes at the time instant $t \geq 0$. By the assumption that the evolution process controlled by the lifetime $\tau$ and the offspring number $\nu$ of living nodes, exact equations have been derived for the generating functions of the probabilities

$$
\mathcal{P}\left\{\mu_{\ell}(t)=n_{\ell}, \mu_{d}(t)=n_{d} \mid \mathcal{S}_{0}\right\}, \quad \text { and } \quad \mathcal{P}\left\{\mu(t)=\mu_{\ell}(t)+\mu_{d}(t)=n \mid \mathcal{S}_{0}\right\}
$$

where $\mathcal{S}_{0}$ indicates that at $t=0$ the tree consisted of a single living node only. To complete the description of the tree dynamics the notion of end-nodes has also been defined. It is remarkable that the average lifetime of living nodes has a role in scaling the time in the generating function equations, only.

The time dependence of the expectation value of the number of living nodes has been analyzed, and as expected, there exist three completely different types of evolution depending on the average number $q_{1}$ of offspring produced by a single living node. If $q_{1}<1$, then the evolution is subcritical, if $q_{1}=1$, then it is critical, while in the case of $q_{1}>1$ is supercritical.

A specific property of the tree evolution has been discovered, namely, it has been proved that the correlation between the numbers of living and dead nodes decreases to zero in subcritical and increases to 1 in supercritical evolution, if the time parameter $Q t$ tends to infinity, but in the case of exactly critical evolution it converges to a fixed value $\sqrt{3} / 2$ which is free of the process parameters.

The stochastic properties of the end-nodes have also been analyzed, and it has been shown that the correlation between the numbers of living and dead end-nodes changes its character rather suddenly at the very beginning of the evolution process. It has been proved that the correlation function is negative just after the start of the process, but it becomes positive very sharply after elapsing a relatively short time. This behavior is the consequence of the fact that the root of the tree can be but a living end-node at the time moment $t=0$.

For the sake of better understanding of the evolution the survival probability of random trees has been investigated, and exact expressions have been derived for this probability in the cases of subcritical, critical, and supercritical evolutions when the generating function of the offspring number $\nu$ was quadratic.

[1] R. Albert and A.-L. Barabási, Rev. Mod. Phys. 74, 47 (2002).

[2] S.N. Dorogovtsev and J.F.F. Mendes, Adv. Phys. 51, 1079 (2002).

[3] S.N. Dorogovtsev and J.F.F. Mendes, Evolution of Networks, (Oxford University Press, New York, 2003 ) p. 241.

[4] T.E. Harris, The theory of Branching Processes, (Springer-Verlag, Berlin-Göttingen-Heidelberg, 1963) p. 33; for the agedependent processes see pp. 121-163.

[5] R. Lyons and Y. Peres, Probability on Trees and Networks, (Cambridge University Press, Cambridge), (to be published).

[6] L. Jánossy, Acta Phys. Acad. Sci. Hung. 2, 289 (1952).

[7] A.T. Bharucha-Reid, Phys. Rev. 96, 751 (1954).

[8] L. Pál, Nuovo Cimento, Suppl. 7, 25 (1958). 
[9] D.G. Kendall, Biometrika, 47, 13 (1960).

[10] G.I. Bell, Ann. Phys. (N.Y.) 21, 243 (1963)

[11] I. Pázsit, Phys. Scr. 59, 344 (1999).

[12] M.M.R. Williams, Ann. Nucl. Energy, 31, 933 (2004).

[13] L. Pál, Prog. Nucl. Energy, 43, 5 (2003).

[14] L. Pál, cond-mat/0205650 (unpublished); cond-mat/0211092 (unpublished); cond-mat/0306540) (unpublished).

[15] B.A. Sevast'yanov, Verzweigungsprozesse, (Akademie-Verlag, Berlin, 1974) p. 28; for the age-dependent processes see pp. 229-309. 